\title{
Giant magneto-spin-Seebeck effect and magnon transfer torques in insulating spin valves
}

Yihong Cheng, Kai Chen, and Shufeng Zhang

Citation: Appl. Phys. Lett. 112, 052405 (2018); doi: 10.1063/1.5018411

View online: $\mathrm{https}: / /$ doi.org/10.1063/1.5018411

View Table of Contents: http://aip.scitation.org/toc/apl/112/5

Published by the American Institute of Physics

\section{Articles you may be interested in}

Spin Seebeck effect and thermal spin galvanic effect in $\mathrm{Ni}_{80} \mathrm{Fe}_{20} / \mathrm{p}$-Si bilayers

Applied Physics Letters 112, 042404 (2018); 10.1063/1.5003008

Spin-orbit torque-induced switching in ferrimagnetic alloys: Experiments and modeling

Applied Physics Letters 112, 062401 (2018); 10.1063/1.5017738

Simple and high-speed polarization-based QKD

Applied Physics Letters 112, 051108 (2018); 10.1063/1.5016931

SAW assisted domain wall motion in $\mathrm{Co} / \mathrm{Pt}$ multilayers

Applied Physics Letters 112, 052402 (2018); 10.1063/1.5000080

Spin-phonon coupling in antiferromagnetic nickel oxide

Applied Physics Letters 111, 252402 (2017); 10.1063/1.5009598

Perpendicular magnetic tunnel junctions with Mn-modified ultrathin MnGa layer

Applied Physics Letters 112, 062402 (2018); 10.1063/1.5002616

\section{Scilight}

Sharp, quick summaries illuminating the latest physics research

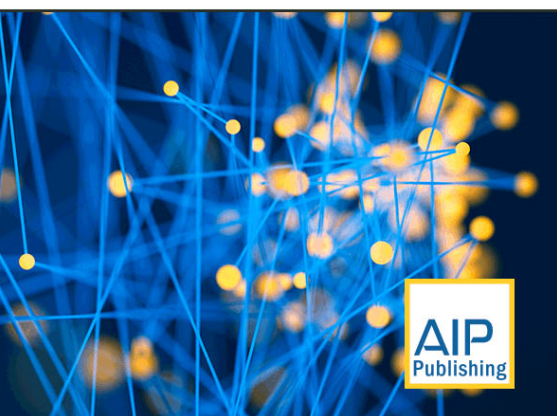




\title{
Giant magneto-spin-Seebeck effect and magnon transfer torques in insulating spin valves
}

\author{
Yihong Cheng, Kai Chen, and Shufeng Zhang ${ }^{\text {a) }}$ \\ Department of Physics, University of Arizona, Tucson, Arizona 85721, USA
}

(Received 6 December 2017; accepted 16 January 2018; published online 31 January 2018)

\begin{abstract}
We theoretically study magnon transport in an insulating spin valve (ISV) made of an antiferromagnetic insulator sandwiched between two ferromagnetic insulator (FI) layers. In the conventional metal-based spin valve, the electron spins propagate between two metallic ferromagnetic layers, giving rise to giant magnetoresistance and spin transfer torque. Here, the incoherent magnons in the ISV serve as angular momentum carriers and are responsible for the angular momentum transport between two FI layers across the antiferromagnetic spacer. We predict two transport phenomena in the presence of the temperature gradient: a giant magneto-spin-Seebeck effect in which the output voltage signal is controlled by the relative orientation of the two FI layers and magnon transfer torque that can be used for switching the magnetization of the FI layers with a temperature gradient of the order of 0.1 Kelvin per nanometer. Published by AIP Publishing.

https://doi.org/10.1063/1.5018411
\end{abstract}

In spintronics, spin current plays an essential role in delivering angular momentum from one layer to another. For example, a conventional spin valve (SV), consisting of two metallic ferromagnets separated by either a metallic or insulating non-magnetic layer, can effectively manipulate the spin current by changing the relative orientations of magnetization of the two magnetic layers. By propagating through two magnetic layers across the non-magnetic spacer, the spin of the conduction electrons interacts with both magnetic layers and leads to two most profound effects: giant magnetoresistance $(\mathrm{GMR})^{1,2}$ and spin transfer torque (STT) ${ }^{3,4}$ For both GMR and STT effects, the magnetic layers must be metallic since the conduction electrons are essential for providing spin transport.

Besides the electron spin, each quasi-particle magnon is also known to have an angular momentum $\hbar$. Magnons are quantized low-energy excitations of magnetic materials. Since magnons are charge neutral, an electric field would not be able to directly drive a magnon current. A temperature gradient, however, can create a magnon current via magnon diffusion from high to low temperature in space. Consequently, the magnon current can transfer its angular momentum to an electron spin current of a heavy metal in contact with the magnetic insulator. Due to the inverse spin Hall effect (ISHE), ${ }^{5}$ this electron spin current generates an electric current or voltage in the heavy metal, which can be directly measured; this is also known as the spin Seebeck effect. ${ }^{6-9}$ Another demonstration of magnons as the angular momentum carriers had recently been achieved in a trilayer $\mathrm{Pt} / \mathrm{YIG} / \mathrm{Pt}$ where the electron spin current in one of the Pt layers can propagate to the other Pt layer mediated by the magnon current in the insulating ferromagnetic YIG layer. ${ }^{10-12}$ In addition to the magnon transport in ferromagnets, magnons in antiferromagnetic materials such as $\mathrm{NiO}$ are also capable of carrying an angular momentum current as reported in trilayer structure $\mathrm{YIG} / \mathrm{NiO} / \mathrm{Pt}^{13-17}$ Besides the above experiments,

a)zhangshu@email.arizona.edu there are a number of existing theoretical studies on magnon transport. ${ }^{18-22}$ Slonczewski ${ }^{23}$ discussed the spin-transfer torque initiated by thermal transport of magnons. In Slonczewski's model, the magnons induced by thermal gradient are to excite conduction electron spins. Thus, the role of the thermal magnons is to initiate spin-dependent electron transport in metal. In our study, the magnon transport in all insulating spin valves does not involve conduction electrons. In terms of STT magnitude, both Slonczewski's work and our work have estimated that the temperature gradient of the order of 1 Kelvin across the interface would generate magnetic torque that is 1-2 orders larger than the conventional STT. However, the thermal gradient is more difficult to maintain in metals than in insulators, and thus, the all-insulator structure we proposed here would be more relevant for experimental verification of magnon transport. All these findings motivate us to investigate an all-insulator spin valve structure, namely, a trilayer consisting of two ferromagnetic insulators (FIs) separated by an antiferromagnetic insulator (AFI), subject to a temperature gradient across the layers, as illustrated in Fig. 1. In this structure, the magnons are expected to travel between two FIs through the AFI spacer and the magnon current would depend on the relative orientations of the two FIs. As a consequence, we show that the magnon valve proposed here displays two distinct phenomena: the giant magneto-spinSeebeck effect (GMSSE) and the magnon transfer torque (MTT). For the GMSSE, the voltage signal detected by the heavy metal (HM) layer attached to one of the FI layers, as shown in Fig. 1, is proportional to the total spin current near the interface of the HM and the thin FI free layer $\left(\mathbf{M}_{F}\right)$ via ISHE. When the magnetization of the two FI layers is parallel (antiparallel), the magnon current induced by the temperature gradient in each FI layer is additive (subtractive), leading to a voltage difference between parallel and antiparallel magnetization alignments. By properly choosing the thickness of the free FI layer, one is able to achieve a 100\% GMSSE ratio (the spin current generated by the antiparallel configuration is zero). For the MTT, when the magnetization of the two FI 


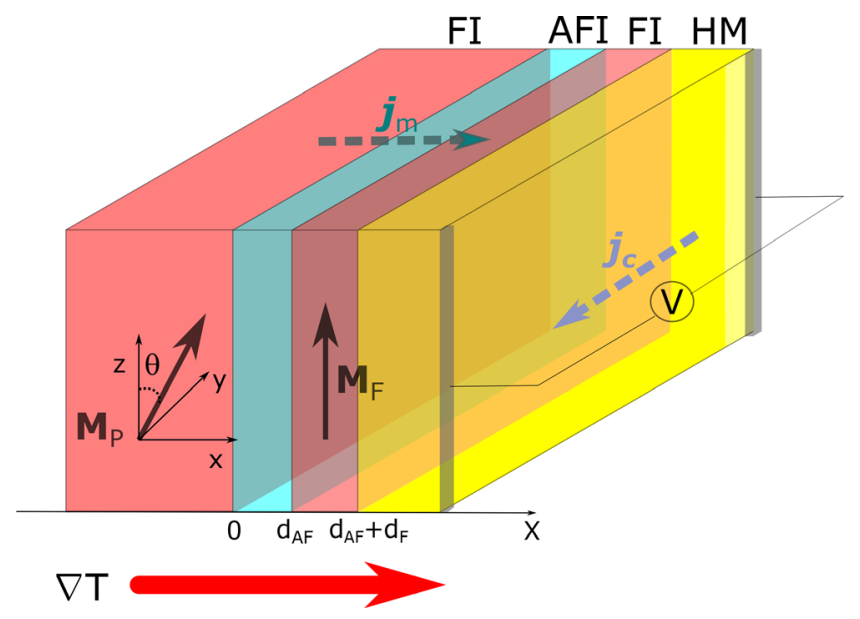

FIG. 1. Schematics of the proposed all-insulating spin valve. Two FI layers are separated by an AFI layer. The attached heavy metal layer is used to read out the magnon current in the ISV. The temperature gradient is applied perpendicular to the layer, and we assume that it is uniform in magnetic layers but zero in the HM layer. In our calculation of GMSSE and MTT, we fixed the direction of the free layer while allowing the direction of the pinned layer to rotate from 0 to $\pi$.

layers is not collinear, the thin FI free layer would absorb the transverse component of the magnon current generated in the thick FI pinned layer $\left(\mathbf{M}_{P}\right)$, similar to the conventional STT phenomenon. If the MTT is sufficiently large, the magnetization of the free FI layer can be switched. We have estimated that a thermal gradient of about 0.1 Kelvin per nanometer could produce a magnon transfer torque of the order of $10^{9} \mathrm{~A} / \mathrm{m}^{2}(\hbar / e)$ (e is the electron charge), a comparable magnitude for the electron current induced STT switching. ${ }^{24-26}$ We present our theory below.

We start by considering a simplest heat profile with a uniform temperature gradient $\nabla_{x} T$ across the insulating spin valve (ISV), i.e., the thermal conductivity is the same for all insulator layers. The temperature gradient in the heavy metal layer is negligibly small. At equilibrium, the average number of magnons in each layer obeys the Bose-Einstein distribution with zero chemical potential. When a thermal gradient exists, magnons would diffuse across the layers and a nonequilibrium magnon distribution would be established. A simplest way to describe the magnon diffusive transport is to introduce an effective magnon chemical potential $\boldsymbol{\mu}_{m}^{\mathrm{F}}$ that characterizes the local magnon spin accumulation. ${ }^{18,19,27,28}$ Similar to the case in ferromagnetic metals, the direction of the magnon chemical potential $\boldsymbol{\mu}_{m}^{\mathrm{F}}$ and magnon current $\mathbf{J}_{m}^{\mathrm{F}}$ is always parallel to the local magnetization $\hat{\mathbf{M}}$, i.e., $\boldsymbol{\mu}_{m}^{\mathrm{F}}$ $=\mu_{m}^{\mathrm{F}} \hat{\mathbf{M}}$ and $\mathbf{J}_{m}^{\mathrm{F}}=J_{m}^{\mathrm{F}} \hat{\mathbf{M}}$ (in units of $\mathbf{J} / \mathrm{m}^{2}$ ), and thus, we may write magnon Ohm's law in a simple scalar form ${ }^{19}$

$$
J_{m}^{\mathrm{F}}=-S_{m}^{x} \nabla_{x} T-\sigma_{m}^{\mathrm{F}} \frac{d \mu_{m}^{\mathrm{F}}}{d x},
$$

where we have defined the magnon thermal conductivity $S_{m}^{x}$ and spin conductivity $\sigma_{m}^{\mathrm{F}}$ as

$$
\begin{aligned}
S_{m}^{x} & =\frac{\hbar \tau_{m}}{(2 \pi)^{3} T} \int d^{3} q v_{q_{x}}^{2} \frac{\varepsilon_{\mathbf{q}}}{k_{B} T} \operatorname{csch}^{2} \frac{\varepsilon_{\mathbf{q}}}{2 k_{B} T} \\
\sigma_{m}^{\mathrm{F}} & =\frac{\hbar \tau_{m}}{(2 \pi)^{3}} \int d^{3} q \frac{v_{q}^{2}}{3} \frac{\partial N_{m}^{0}(\mathbf{q})}{\partial \varepsilon_{\mathbf{q}}}
\end{aligned}
$$

where $\mathbf{v}_{\mathbf{q}}$ is the magnon velocity, $\tau_{m}$ is the magnon relaxation time, $\varepsilon_{\mathbf{q}}$ is the magnon energy, and $N_{m}^{0}(\mathbf{q})=\left[\exp \left(\beta \varepsilon_{\mathbf{q}}\right)-1\right]^{-1}$ is the equilibrium magnon distribution. The magnon accumulation in Eq. (1) satisfies the diffusion equation, $d^{2} \mu_{m}^{\mathrm{F}} / d x^{2}$ $=\mu_{m}^{\mathrm{F}} /\left(\lambda_{m}^{\mathrm{F}}\right)^{2}$, where $\lambda_{m}^{\mathrm{F}}$ is the magnon diffusion length.

For the AFI, magnon diffusion is generally more complicated. If the AFI is a single crystal with collinear magnetic moments for two sublattices, the magnon spectra contain two degenerate branches with each magnon carrying either $\hbar$ or $-\hbar$ with respect to the order parameter (the staggered magnetization direction). For a polycrystalline AFI layer, there are multiple directions for the order parameter and the description of the magnon transport would be very cumbersome and difficult. Here, we shall consider a simple realization in which the order parameter of the AFI may be distributed randomly; this is certainly an oversimplification for modelling a polycrystalline AFI, but the problem becomes trackable. In this picture, the magnon propagation in the AFI is similar to the electron spin transport in a nonmagnetic metal in which the spin or magnon currents can be oriented in any direction dictated by the spin/magnon injection from the neighboring magnetic layer. Thus, magnon Ohm's law in the AFI may be similarly defined as

$$
J_{m}^{\mathrm{AF}}=-\sigma_{m}^{\mathrm{AF}} \frac{d \mu_{m}^{\mathrm{AF}}}{d x},
$$

where $\sigma_{m}^{\mathrm{AF}}$ is the magnon spin conductivity of AFI; for a collinear $\mathrm{AF}$ spin structure, $\sigma_{m}^{\mathrm{AF}}$ has already been calculated. ${ }^{29}$ It is noted that the AFI has no drift term associated with the temperature gradient since the magnon current from the two degenerated magnon spectra cancels out; ${ }^{29-31}$ this is analogous to the electron spin transport where an electric field induces a spin current drift term in ferromagnetic metal but not in non-magnetic metal. The magnon chemical potential in the AFI layer also satisfies the diffusion equation, $d^{2} \mu_{m}^{\mathrm{AF}} / d x^{2}=\mu_{m}^{\mathrm{AF}} /\left(\lambda_{m}^{\mathrm{AF}}\right)^{2}$, where $\lambda_{m}^{\mathrm{AF}}$ is the magnon diffusion length of the AFI layer. Since $\boldsymbol{\mu}_{m}^{\mathrm{AF}}$ can take any direction, the general solution of $\mu_{m}^{\mathrm{AF}}$ is

$$
\boldsymbol{\mu}_{m}^{\mathrm{AF}}(x)=\mathbf{A} \exp \left(\frac{x}{\lambda_{m}^{\mathrm{AF}}}\right)+\mathbf{B} \exp \left(-\frac{x}{\lambda_{m}^{\mathrm{AF}}}\right),
$$

where $\mathbf{A}$ and $\mathbf{B}$ are the vectorial constants of the integrations. Clearly, when the magnetization $\mathbf{M}_{P}$ and $\mathbf{M}_{F}$ are not collinear, the direction of $\boldsymbol{\mu}_{m}^{\mathrm{AF}}$ is parallel to neither $\mathbf{M}_{P}$ nor $\mathbf{M}_{F}$. The constant vectors $\mathbf{A}$ and $\mathbf{B}$ would be determined by the boundary conditions given below.

The first set of boundary conditions involves the longitudinal magnon chemical potential and magnon current. When neglecting the interfacial spin memory loss, the longitudinal spin current is continuous across the FI/AFI interfaces, i.e.,

$$
J_{m}^{\mathrm{F}}\left(0^{-}\right)=\hat{\mathbf{M}}_{P} \cdot \mathbf{J}_{m}^{\mathrm{AF}}\left(0^{+}\right) ; \quad J_{m}^{\mathrm{F}}\left(d_{\mathrm{AF}}^{+}\right)=\hat{\mathbf{M}}_{F} \cdot \mathbf{J}_{m}^{\mathrm{AF}}\left(d_{\mathrm{AF}}^{-}\right) .
$$

The magnon current across the interfaces is also related to the difference in the magnon chemical potential at the two sides of the interface

$$
J_{m}(0)=\frac{G_{\mathrm{A} / \mathrm{F}}^{\|}}{2 \pi}\left[\mu_{m}^{\mathrm{F}}\left(0^{-}\right)-\boldsymbol{\mu}_{m}^{\mathrm{AF}}\left(0^{+}\right) \cdot \hat{\mathbf{M}}_{P}\right]
$$


where $G_{\mathrm{A} / \mathrm{F}}^{\|}$is the conductance addressing spin convertance efficiency across interfaces. ${ }^{20,22} \mathrm{~A}$ similar expression at the interface $x=d_{\mathrm{AF}}$ can also be written.

The second set of boundary conditions relates the magnon chemical potential and magnon current perpendicular to the local magnetization of the FI layers. Similar to the ferromagnetic metal case where the electron spin chemical potential and spin current transverse to the magnetization direction are zero, ${ }^{32,33}$ the transverse magnon chemical potential and magnon current vanish in the FI layers as well. Thus, the transverse magnon current and chemical potential at the boundaries are

$$
\hat{\mathbf{M}}_{P} \times\left[\hat{\mathbf{M}}_{P} \times \mathbf{J}_{m}^{\mathrm{AF}}\left(0^{+}\right)\right]=-\frac{G_{\mathrm{A} / \mathrm{F}}^{\perp}}{2 \pi} \hat{\mathbf{M}}_{P} \times\left[\hat{\mathbf{M}}_{P} \times \boldsymbol{\mu}_{m}^{\mathrm{AF}}\left(0^{+}\right)\right]
$$

and the similar expression for the interface at $x=d_{\mathrm{AF}}$. The thermally driven interfacial magnon conductance for the transverse component $G_{\mathrm{A} / \mathrm{F}}^{\perp}$ is analogous to the mixing conductance of electron spin, ${ }^{34,35}$ and it differs from the longitudinal one $G_{\mathrm{A} / \mathrm{F}}^{\|}$. Finally, the boundary conditions at the interface of the FI and HM layers involve the conversion between the magnon current and the electron spin current of the HM layer; it has been previously formulated in Ref. 22 .

With these boundary conditions, the magnon chemical potential and magnon current are determined in each layer. In the calculation shown below, we shall take the thickness of the pinned layer much larger than the magnon diffusion length $\lambda_{m}^{\mathrm{F}}$ and the thickness of the antiferromagnetic layer much less than the magnon diffusion length $\lambda_{m}^{\mathrm{AF}}$.

Figure 2 shows the thickness dependence of the giant magneto-spin-Seebeck spin current signal for the two magnetic layers in parallel and antiparallel configurations. In the parallel alignment, the magnon current at the HM interface is the sum of the magnon sources from both FI layers. At a small thickness of the free layer, the main contribution is from the pinned layer whose magnon current travels through two AFI/FI interfaces. As the thickness increases, the contribution from the free layer increases and that from the pinned layer decreases because the magnon current from it is further away from the FI/HM interface with a higher probability to be scattered by the free layer. To see the relative contribution of the magnon current from the two FI layers, we turn off the temperature gradient either in the pinned or in the free layer, as shown in the inset of Fig. 2(a). The decrease in the magnon current from the pinned layer is steeper than the increase from the free layer for a small thickness of the free layer. For the magnon current generated in the fixed layer, the exponential decaying length involves multiple interface scattering in addition to the scattering in the free layer, while the magnon current generated in the free layer linearly increases with its thickness; this explains the initial reduction in the total magnon current at a small thickness shown in Fig. 2(a). When the thickness becomes larger, the contribution from the free layer is more important and eventually dominates the entire magnon current for the thickness much larger than the magnon diffusion length. For the antiparallel alignment, the magnon current from the pinned layer is always opposite to that from the free layer. As the thickness of the free layer
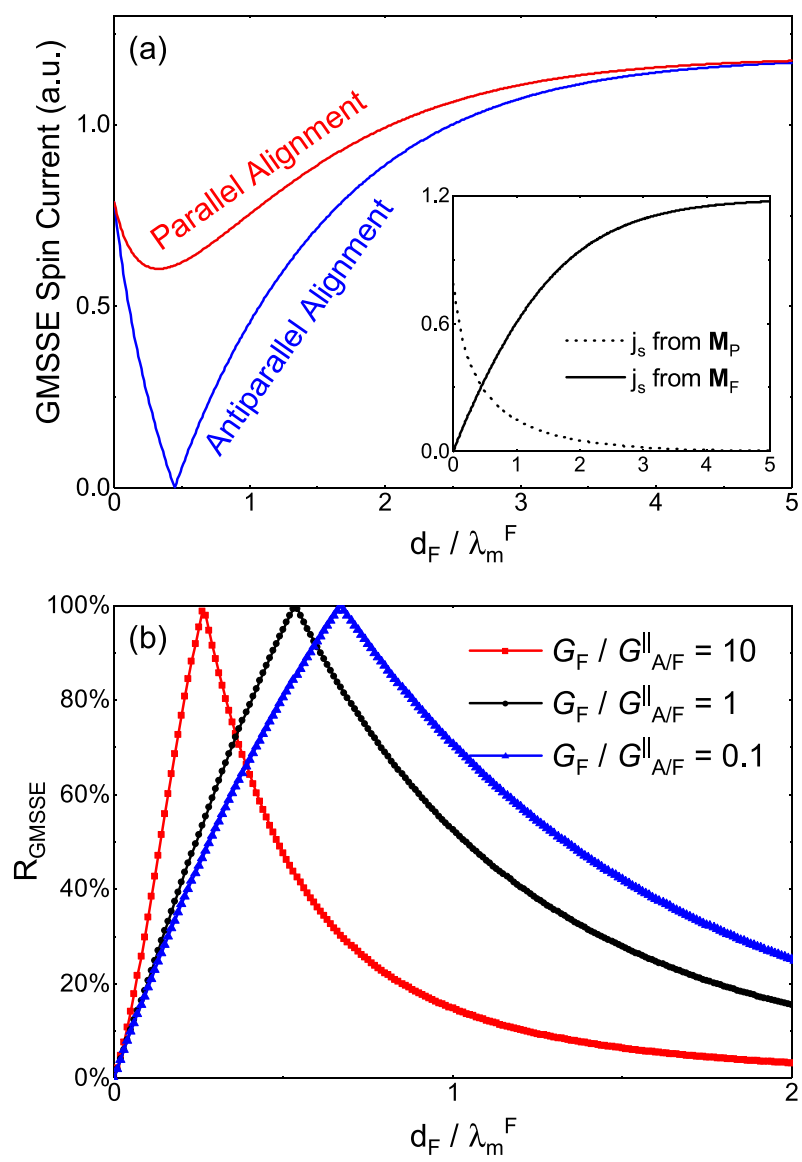

FIG. 2. Thickness dependence of the GMSSE. (a) The GMSSE spin current (arbitrary unit) is shown for the parallel (red solid line) and antiparallel (blue solid line) aligned FI layers and (b) the GMSSE ratio. The parameters used in the figure are: magnon spin conductance for the FI layer $G_{\mathrm{F}} \equiv \sigma_{m}^{\mathrm{F}} / \lambda_{m}^{\mathrm{F}}=2.1 \times 10^{18} \mathrm{~m}^{-2}$, electron spin conductance for the NM layer $G_{\mathrm{N}}=7.7 \times 10^{18} \mathrm{~m}^{-2}$, and spin conductance for the FI/NM interface $G_{\mathrm{F} / \mathrm{N}}=0.3 \times 10^{18} \mathrm{~m}^{-2}$.

increases, the magnitude of the total magnon current first decreases due to partial cancellation of the contributions from the two magnetic layers. At a certain critical thickness $d_{\mathrm{cr}}$, the magnon currents from two layers are exactly cancelled and the total magnon current becomes identically zero. In this case, we completely achieved an off-state in which the ISHE voltage is zero. If we define a giant magneto-spin-Seebeck ratio by $R_{\mathrm{GMSSE}}=\left(j_{s}^{P}-j_{s}^{A P}\right) / j_{s}^{P}$, where $j_{s}^{P}$ and $j_{s}^{A P}$ are the magnon spin current magnitudes for parallel and antiparallel alignments of the FI layers, we arrive at the thickness dependence of $R_{\mathrm{GMSSE}}$ shown in Fig. 2(b). It is noted that the $100 \%$ giant magneto-spin-Seebeck effect is always possible by choosing a proper thickness of the free layer.

We next calculate the magnon transfer torque $\tau_{\text {MTT }}$ on the free layer. At the interface between AFI and the free layer, the transverse part of the spin current of the AFI layer is unable to penetrate into the free layer. Similar to the conventional spin transfer torque, one may identify the MTT as the transverse magnon current density at the AFI side of the interface, namely, $\tau_{\mathrm{MTT}}=\hat{\mathbf{M}}_{F} \times\left(\mathbf{J}_{m}^{\mathrm{AF}}\left(d_{\mathrm{AF}}^{-}\right) \times \hat{\mathbf{M}}_{F}\right)$. In Fig. 3, we show the MTT for the two perpendicularly aligned AFI layers as a function of the magnon diffusion length $\lambda_{m}^{\mathrm{F}}$ for $d_{\mathrm{F}}=3$ $\mathrm{nm}$ and for several temperature gradients. The nearly linear relation between the MTT and the diffusion length is due to the fact that we have chosen the pinned layer infinitely thick 


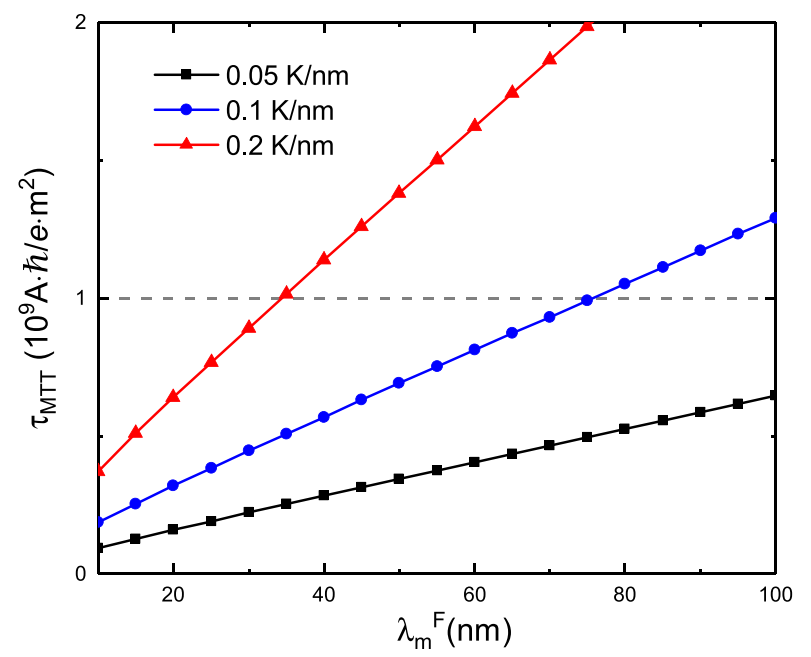

FIG. 3. The magnon transfer torque as a function of the magnon diffusion length at room temperature. The parameters used in the figure are $G_{\mathrm{A} / \mathrm{F}}^{\perp}=1$ $\times 10^{17} \mathrm{~m}^{-2}, G_{\mathrm{A} / \mathrm{F}}^{\|}=1 \times 10^{18} \mathrm{~m}^{-2}, G_{\mathrm{F}}, G_{\mathrm{N}}$, and $G_{\mathrm{F} / \mathrm{N}}$ are the same as used in Fig. 2.

such that the spin current flowing from the pinned layer to the free layer scales as $k_{B} \nabla_{x} T \lambda_{m}^{\mathrm{F}}{ }^{22}$ If the thickness is finite, the linear relation for a large $\lambda_{m}^{\mathrm{F}}$ will no longer be held. In determining the absolute value of the MTT in Fig. 3, we have used the following plausible parameters relevant to typical magnetic insulators of $\mathrm{YIG}^{28}$ and the $\mathrm{YIG} / \mathrm{NiO}$ interface: $T_{C}=550 \mathrm{~K}$, $a_{\mathrm{YIG}}=1.39 \mathrm{~nm}$, quadratic dispersion relation $\varepsilon_{\mathbf{q}}=A q^{2}+\Delta_{g}$ with exchange stiffness $A=6 \mathrm{meV} \cdot \mathrm{nm}^{2}$ and magnon gap $\Delta_{g}=10^{-6} \mathrm{eV}$, and $G_{\mathrm{YIG} / \mathrm{NiO}}^{\|}=1 \times 10^{18} \mathrm{~m}^{-2}$.

It is interesting to compare the absolute values of the MTT with the STT. In the conventional STT devices, one needs a critical electric current density on the order of $J_{c} \sim 10^{10} \mathrm{~A} / \mathrm{m}^{224-26}$ to switch a metallic ferromagnetic layer. Assuming that the electric current is $100 \%$ spin polarized, the angular momentum current would be $(\hbar / e) J_{c}$. For switching an insulating ferromagnet which typically has a smaller Gilbert damping parameter and lower saturation magnetization, we would expect an order of magnitude smaller critical angular momentum current. In Fig. 3, the horizontal dotted line represents this hypothetical critical value for the magnetization switching. Thus, we estimate that a temperature gradient of the order of $0.1 \mathrm{~K} / \mathrm{nm}$ for $\lambda_{m}^{\mathrm{F}}=80 \mathrm{~nm}^{12,19}$ is capable of achieving the magnetization switching.

If we write the MTT in the vector form, $\tau_{\mathrm{MTT}}$ $=\tau_{\mathrm{MTT}}^{0} \hat{\mathbf{M}}_{F} \times\left(\hat{\mathbf{M}}_{P} \times \hat{\mathbf{M}}_{F}\right)$, the magnitude $\tau_{\mathrm{MTT}}^{0}$ is the transverse component of $\mathbf{J}_{m}^{\mathrm{AF}}\left(d_{\mathrm{AF}}^{-}\right)$which depends on the relative angle between two magnetic layers. In Fig. 4, we show the angular dependence of the MTT; this behavior is similar to that of the spin torque in the conventional metal-based SV. ${ }^{33}$

We emphasize some of the key features in our proposed magnon valves. First, the GMSSE and the MTT phenomena proposed here are strongly temperature dependent. Since the number of magnons in the FI and AFI is much robust at high temperature, we expect the predicted GMSSE and MTT to be diminished at low temperature. This temperature dependence is rather general for all magnon driven transport phenomena and has been reported in various experiments related to magnon transport. Second, the modeling of the magnon transport in AFI is oversimplified. We have used a simple

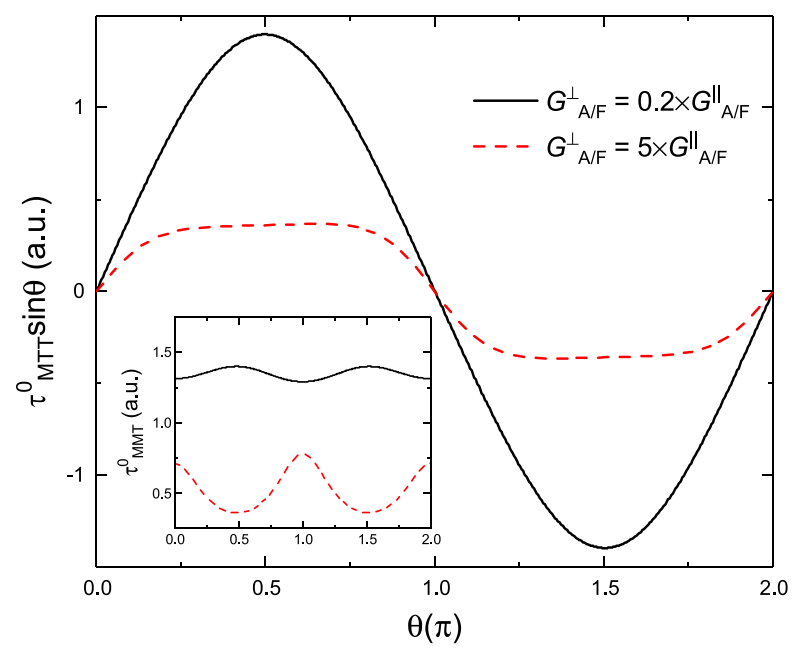

FIG. 4. The angular dependence of the magnon transfer torque, where $\theta$ is the angle between the magnetization of $\mathbf{M}_{P}$ and $\mathbf{M}_{F}$ layers. In the inset, we show the angular dependence of the MTT without $\sin \theta$.

magnon dispersion relation with an ideal collinear sublattice AF Hamiltonian, and we further assume that the AFI can transmit any direction of the spin current. These simplifications lead to uncertainty in the estimation of the MTT. More specific models relevant to experimental materials should be used to give a better estimation. Third, the interaction at the interface between the AFI and FI could have profound effects. The exchange bias and coercivity enhancement would complicate the interface magnon conductance compared to that for an ideal atomically flat interface, and furthermore, other effects of the MTT on the magnetization switching could emerge.

In summary, we have proposed a magnon valve structure based on insulating magnetic materials. Similar to the giant magnetoresistance and the spin transfer torque in the conventional spin valve, we predict two phenomena in the giant magneto-spin-Seebeck effect and magnon transfer torque. From the view point of fundamental study of spin transport, the ISV offers an ideal platform for magnon angular momentum transport, without the complications of metalbased SV where both magnons and electrons are involved in the transport. In terms of device application, the ISV guarantees zero Joule energy lost for minimizing power consumption and lower damping for faster magnetization switching. On the other hand, controlling the direction of bi-polar heat flow and determining the temperature gradient at the nanometer scale for reversibly switching magnetization may present a challenge for device designs.

This work was partially supported by the U.S. National Science Foundation under Grant No. ECCS-1708180.

${ }^{1}$ M. N. Baibich, J. M. Broto, A. Fert, F. N. Van Dau, F. Petroff, P. Etienne, G. Creuzet, A. Friederich, and J. Chazelas, Phys. Rev. Lett. 61, 2472 (1988).

${ }^{2}$ G. Binasch, P. Grnberg, F. Saurenbach, and W. Zinn, Phys. Rev. B 39, 4828 (1989).

${ }^{3}$ J. C. Slonczewski, J. Magn. Magn. Mater. 159, L1 (1996).

${ }^{4}$ L. Berger, Phys. Rev. B 54, 9353 (1996).

${ }^{5}$ E. Saitoh, M. Ueda, H. Miyajima, and G. Tatara, Appl. Phys. Lett. 88, 182509 (2006).

${ }^{6}$ K.-I. Uchida, H. Adachi, T. Ota, H. Nakayama, S. Maekawa, and E. Saitoh, Appl. Phys. Lett. 97, 172505 (2010). 
${ }^{7}$ S. Y. Huang, W. G. Wang, S. F. Lee, J. Kwo, and C. L. Chien, Phys. Rev. Lett. 107, 216604 (2011).

${ }^{8}$ M. Weiler, M. Althammer, F. D. Czeschka, H. Huebl, M. S. Wagner, M. Opel, I.-M. Imort, G. Reiss, A. Thomas, R. Gross, and S. T. B. Goennenwein, Phys. Rev. Lett. 108, 106602 (2012).

${ }^{9}$ K. Uchida, T. Nonaka, T. Kikkawa, Y. Kajiwara, and E. Saitoh, Phys. Rev. B 87, 104412 (2013).

${ }^{10}$ L. J. Cornelissen, J. Liu, R. A. Duine, J. B. Youssef, and B. J. van Wees, Nat. Phys. 11, 1022 (2015).

${ }^{11}$ J. Li, Y. Xu, M. Aldosary, C. Tang, Z. Lin, S. Zhang, R. Lake, and J. Shi, Nat. Commun. 7, 10858 (2016).

${ }^{12}$ H. Wu, C. H. Wan, X. Zhang, Z. H. Yuan, Q. T. Zhang, J. Y. Qin, H. X. Wei, X. F. Han, and S. Zhang, Phys. Rev. B 93, 060403 (2016).

${ }^{13}$ H. Wang, C. Du, P. C. Hammel, and F. Yang, Phys. Rev. Lett. 113, 097202 (2014).

${ }^{14}$ C. Hahn, G. D. Loubens, V. V. Naletov, J. B. Youssef, O. Klein, and M. Viret, Europhys. Lett. 108, 57005 (2014).

${ }^{15}$ W. Lin, K. Chen, S. Zhang, and C. Chien, Phys. Rev. Lett. 116, 186601 (2016).

${ }^{16}$ Z. Qiu, J. Li, D. Hou, E. Arenholz, A. T. NDiaye, A. Tan, K-i. Uchida, K. Sato, S. Okamoto, Y. Tserkovnyak, Z. Q. Qiu, and E. Saitoh, Nat. Commun. 7, 12670 (2016).

${ }^{17}$ Y. M. Hung, C. Hahn, H. Chang, M. Wu, H. Ohldah, and A. D. Kent, AIP Adv. 7, 055903 (2017).

${ }^{18}$ S. S.-L. Zhang and S. Zhang, Phys. Rev. Lett. 109, 096603 (2012).

${ }^{19}$ S. M. Rezende, R. L. Rodrguez-Surez, R. O. Cunha, A. R. Rodrigues, F. L. A. Machado, G. A. Fonseca Guerra, J. C. Lopez Ortiz, and A. Azevedo, Phys. Rev. B 89, 014416 (2014).
${ }^{20}$ L. J. Cornelissen, K. J. H. Peters, G. E. W. Bauer, R. A. Duine, and B. J. van Wees, Phys. Rev. B 94, 014412 (2016).

${ }^{21}$ S. A. Bender and Y. Tserkovnyak, Phys. Rev. B 93, 064418 (2016).

${ }^{22}$ K. Chen, W. Lin, C. L. Chien, and S. Zhang, Phys. Rev. B 94, 054413 (2016).

${ }^{23}$ J. C. Slonczewski, Phys. Rev. B 82, 054403 (2010).

${ }^{24}$ Y. Huai, F. Albert, P. Nguyen, M. Pakala, and T. Valet, Appl. Phys. Lett. 84, 3118 (2004).

${ }^{25}$ Z. Diao, D. Apalkov, M. Pakala, Y. Ding, A. Panchula, and Y. Huai, Appl. Phys. Lett. 87, 232502 (2005).

${ }^{26}$ Z. Diao, A. Panchula, Y. Ding, M. Pakala, S. Wang, Z. Li, D. Apalkov, H. Nagai, A. Driskill-Smith, L.-C. Wang, E. Chen, and Y. Huai, Appl. Phys. Lett. 90, 132508 (2007).

${ }^{27}$ S. A. Bender, R. A. Duine, and Y. Tserkovnyak, Phys. Rev. Lett. 108, 246601 (2012).

${ }^{28}$ S. S.-L. Zhang and S. Zhang, Phys. Rev. B 86, 214424 (2012).

${ }^{29}$ S. M. Rezende, R. L. Rodrguez-Surez, and A. Azevedo, Phys. Rev. B 93, 014425 (2016).

${ }^{30}$ F. Keffer, H. Kaplan, and Y. Yafet, Am. J. Phys. 21, 250 (1953).

${ }^{31}$ Y. Ohnuma, H. Adachi, E. Saitoh, and S. Maekawa, Phys. Rev. B 87, 014423 (2013).

${ }^{32}$ M. D. Stiles and A. Zangwill, Phys. Rev. B 66, 014407 (2002).

${ }^{33}$ J. Barnaś, A. Fert, M. Gmitra, I. Weymann, and V. K. Dugaev, Phys. Rev. B 72, 024426 (2005).

${ }^{34}$ A. Brataas, Y. V. Nazarov, and G. E. W. Bauer, Phys. Rev. Lett. 84, 2481 (2000).

${ }^{35}$ Y. Tserkovnyak, A. Brataas, and G. E. W. Bauer, Phys. Rev. Lett. 88, 117601 (2002). 